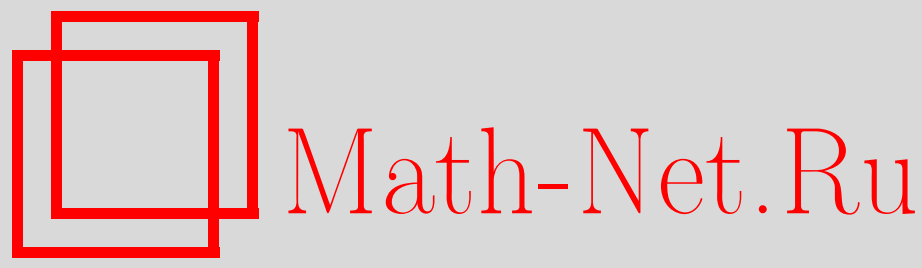

Н. Х. Усеинов, Квазиклассические функции Грина магнитных точечных контактов, ТМФ, 2015, том 183, номер 2, 301-311

DOI: https://doi.org/10.4213/tmf8786

Использование Общероссийского математического портала Math-Net.Ru подразумевает, что вы прочитали и согласны с пользовательским соглашением http://www.mathnet.ru/rus/agreement

Параметры загрузки:

IP : 54.89.56.158

26 апреля 2023 г., 17:54:29

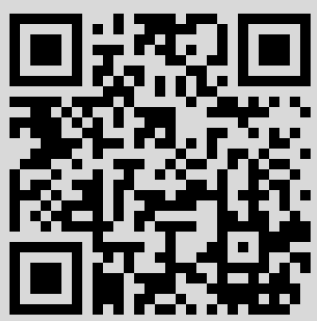




\title{
КВАЗИКЛАССИЧЕСКИЕ ФУНКЦИИ ГРИНА МАГНИТНЫХ ТОЧЕЧНЫХ КОНТАКТОВ
}

\begin{abstract}
Предложен метод построения квазиклассических симметричных и антисимметричных функций Грина магнитных точечных контактов с однородной намагниченностью различных ферромагнитных электродов. Полученная антисимметричная функция Грина позволяет исследовать баллистический и диффузный транспорт электронов через магнитный контакт с учетом неоднородности электрохимического потенциала.
\end{abstract}

Ключевые слова: квазиклассическая функция Грина, магнитная гетероструктура, магнитный точечный контакт, обыкновенные дифференциальные уравнения.

DOI: $10.4213 / \operatorname{tmf} 8786$

\section{1. ВВЕДЕНИЕ}

Известно, что эффективным методом исследования задачи электронного транспорта в теории проводимости сверхпроводников и нормальных металлов являются квазиклассические уравнения для функций Грина (см., например, [1]). В соответствующей формулировке квазиклассическая теория явлений переноса в микроконтактах с сужениями, основанная на дифференциальных уравнениях для функций Грина, была развита в работе Зайцева [2]. В этой работе была получена система квазиклассических уравнений, позволяющая исследовать неравновесные и нестационарные явления проводимости в структурах, содержащих одну или несколько границ между металлами. Эта система содержит матричные функции Грина, позволяющие описывать падающие на границы и отраженные от границ волны электронов. Эти функции Грина по обе стороны от границы сшиваются с помощью граничных условий, которые выведены для произвольной формы и произвольной прозрачности потенциального барьера. Квазиклассическая теория, которая описывает явления с характерными длинами, бо́льшими, чем длины волны Ферми $k_{\mathrm{F}}^{-1}$, и частотами ниже, чем частота Ферми $\varepsilon_{\mathrm{F}} / \hbar$, также может быть математическим базисом для исследования термодинамических и транспортных свойств магнитных гетероструктур. Построение квазиклассической функции Грина таких (магнитных)

* Институт физики, Казанский Приволжский федеральный университет, Казань, Россия. E-mail: nuseinov@mail.ru 
гетероструктур и точечных контактов неоднократно проводилось в рамках различных моделей [3]-[9]. В частности, авторы работы [4], развивая квазиклассическую модель для функций Грина и используя граничные условия Зайцева [2], получили выражения для проводимостей при параллельной и антипараллельной ориентациях намагниченностей ферромагнитного гомоконтакта. В рамках этой модели были исследованы зависимости магнитосопротивления магнитного точечного контакта как функции отношения его радиуса $a$ к длине свободного пробега $l$ электрона проводимости. Предложенная схема вычисления функций Грина основывалась на интерполяции слагаемых в функциях Грина в двух режимах: диффузном $(l<a)$ и баллистическом $(l>a)$. При построении функции Грина ферромагнитного гетероконтакта (см., например, [7], [9]) приходится решать задачи, обусловленные геометрией исследуемой системы, проводить сшивки спин-поляризованных каналов проводимости и вычислять коэффициент прохождения энергетического барьера. Кроме того, в результате несоответствия между зонами проводимости и магнитными свойствами контактирующих металлов появляется граничное сопротивление [8], связанное с кривизной электрохимических потенциалов (см. рис. 1б). Все это делает процедуру построения квазиклассической функции Грина магнитных точечных контактов нетривиальной задачей даже в простейшей модели, когда в намагниченных берегах контакта электроны описываются как свободные с обменным расщеплением спиновых подзон.

В упомянутой работе [4] квазиклассические функции Грина удовлетворяют системе линейных дифференциальных уравнений (аналогичных уравнениям Больцмана в $\tau$-приближении)

$$
\begin{array}{r}
l_{z, \alpha} \frac{\partial g_{\mathrm{a}, \alpha}(z, \boldsymbol{\rho}, t)}{\partial z}+\mathbf{l}_{\|, \alpha} \frac{\partial g_{\mathrm{s}, \alpha}(z, \boldsymbol{\rho}, t)}{\partial \boldsymbol{\rho}}+g_{\mathrm{s}, \alpha}(z, \boldsymbol{\rho}, t)-\left\langle g_{\mathrm{s}, \alpha}\right\rangle=0 \\
l_{z, \alpha} \frac{\partial g_{\mathrm{s}, \alpha}(z, \boldsymbol{\rho}, t)}{\partial z}+\mathbf{l}_{\|, \alpha} \frac{\partial g_{\mathrm{a}, \alpha}(z, \boldsymbol{\rho}, t)}{\partial \boldsymbol{\rho}}+g_{\mathrm{a}, \alpha}(z, \boldsymbol{\rho}, t)=0
\end{array}
$$

где $g_{\mathrm{s}(\mathrm{a}), \alpha}(z, \boldsymbol{\rho}, t)$ - симметричная (антисимметричная) функция Грина, $\mathbf{l}_{\|, \alpha}-$ вектор, определяющий длину свободного пробега электрона в плоскости контакта (см. рис. 1), абсолютная величина этого вектора определяется геометрией: $l_{\|, \alpha}^{2}=l_{\alpha}^{2}-l_{z, \alpha}^{2}$, где $l_{z, \alpha}=l_{\alpha} \cos \theta$ - проекция на ось $z, \alpha$ - спиновый индекс. Угловые скобки в первом уравнении (1) означают усреднение по телесному углу:

$$
\left\langle g_{\mathrm{s}, \alpha}\right\rangle=\frac{1}{2 \pi} \int_{0}^{\pi / 2} \int_{0}^{2 \pi} g_{\mathrm{s}, \alpha} \sin \theta d \theta d \varphi .
$$

Симметричная и антисимметричная функции Грина определяются по отношению к проекции $n_{z}=p_{z, \alpha} / p_{\mathrm{F}, \alpha}$ импульса Ферми $p_{\mathrm{F}, \alpha}$ на ось $z$ :

$$
g_{\mathrm{a}(\mathrm{s}), \alpha}(z, \boldsymbol{\rho}, t)=\frac{1}{2}\left[g_{\alpha}\left(n_{z}, z, \boldsymbol{\rho}, t\right) \mp g_{\alpha}\left(-n_{z}, z, \boldsymbol{\rho}, t\right)\right] .
$$

Граничные условия задачи в точке $z=0$, выведенные в работе Зайцева [2], имеют вид

$$
\begin{aligned}
& g_{\mathrm{a}, \alpha}^{\mathrm{L}}(0)=g_{\mathrm{a}, \alpha}^{\mathrm{R}}(0)= \begin{cases}g_{\mathrm{a}, \alpha}(0), & p_{\|, \alpha}<p_{\mathrm{F}, \alpha}^{\mathrm{L}}, p_{\mathrm{F}, \alpha}^{\mathrm{R}}, \\
0, & p_{\|, \alpha}>\min \left[p_{\mathrm{F}, \alpha}^{\mathrm{L}}, p_{\mathrm{F}, \alpha}^{\mathrm{R}}\right],\end{cases} \\
& 2 R_{\alpha} g_{\mathrm{a}, \alpha}(0)=D_{\alpha}\left(g_{\mathrm{s}, \alpha}^{\mathrm{L}}(0)-g_{\mathrm{s}, \alpha}^{\mathrm{R}}(0)\right) \text {, }
\end{aligned}
$$




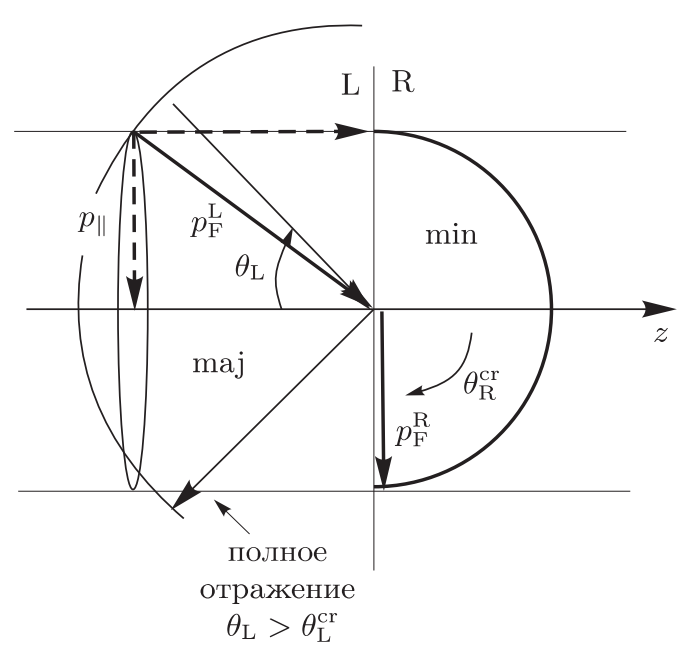

a

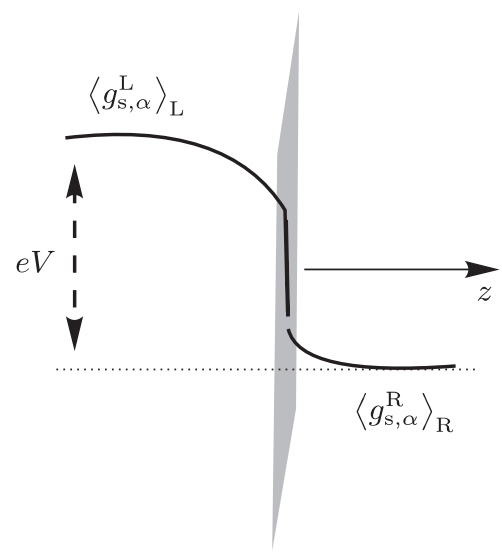

б

Рис. 1. а - Схематическое изображение точечного контакта двух ферромагнитных металлов. Полуокружности соответствуют поверхностям Ферми для спиновых подзон меньшинства ( $\mathrm{min})$ электронов в правом $(\mathrm{R})$ и большинства (maj) электронов в левом (L) электродах. Жирные стрелки показывают критический угол $\theta_{\mathrm{L}}^{\mathrm{cr}}$ падения электрона на контакт. Тонкие стрелки обозначают (падение) отражение электрона при угле падения больше критического, $\theta_{\mathrm{L}}>\theta_{\mathrm{L}}^{\text {cr }}$, при котором возникает полное отражение электронов. Штриховые стрелки обозначают параллельную и перпендикулярную к интерфейсу компоненты импульса Ферми. б - Схематическая иллюстрация кривизны и скачка электрохимического потенциала на границе. Отметим, что скачок электрического потенциала (не измеримый вольтметром) имеется уже в отсутствие тока.

где $p_{\|, \alpha}-$ проекция спин-зависимого импульса Ферми $p_{\mathrm{F}, \alpha}^{\mathrm{L}(\mathrm{R})}$ на плоскость контакта, верхними индексами $\mathrm{L}$ и $\mathrm{R}$ обозначены левая и правая стороны контакта, $D_{\alpha}$ и $R_{\alpha}=1-D_{\alpha}$ - квантово-механические коэффициенты прохождения и отражения области контакта, зависящие от $p_{\mathrm{F}, \alpha}^{\mathrm{L}(\mathrm{R})}$, спинового канала проводимости и угла подлета электрона к плоскости контакта (см. рис. 1а). Смысл граничных условий (4) заключается в равенстве чисел электронов, влетающих в контакт и вылетающих из него. Граничное условие (5) свидетельствует о том, что плотность тока $j_{\alpha}$, которая определяется функцией $\left\langle g_{\mathrm{a}, \alpha}\right\rangle$, пропорциональна разности потенциалов $e V \propto\left(\left\langle g_{\mathrm{s}, \alpha}^{\mathrm{L}}\right\rangle-\left\langle g_{\mathrm{s}, \alpha}^{\mathrm{R}}\right\rangle\right)$, где $V$ - приложенное напряжение к берегам контакта или напряжение, возникающее за счет контактной разности потенциалов. Граничное условие (5) здесь играет роль закона Ома вдоль квазиклассической траектории движения электрона. Отметим также, что соотношения (4) и (5) подчиняются условию сохранения компоненты импульса, лежащей в плоскости контакта:

$$
p_{\|, \alpha}=p_{\mathrm{F}, \alpha}^{\mathrm{L}} \sin \theta_{\mathrm{L}}=p_{\mathrm{F}, \alpha}^{\mathrm{R}} \sin \theta_{\mathrm{R}}
$$




\section{2. ПОСТАНОВКА ЗАДАЧИ}

В работе [10] мы определили квазиклассические функции Грина магнитного точечного гетероконтакта. Это позволило вычислить спин-поляризованные проводимости гетероконтакта при параллельной и антипараллельной намагниченности ферромагнитных электродов. В данной работе мы рассмотрим более общую задачу: нахождение симметричной и антисимметричной функций Грина гетероконтакта, где ферромагнитные электроды могут быть разделены (немагнитным) проводящим или диэлектрическим слоем. Импульсы Ферми спиновых подзон и спин-зависимые от спина длины свободного пробега электронов проводимости в контактирующих ферромагнетиках будут произвольными. Предложенная схема вычисления основывается на интерполяции слагаемых в функциях Грина в двух режимах: диффузном $(l<a)$ и баллистическом $(l>a)$. Кроме того, мы учтем градиентные слагаемые в разложении функции Грина, которые позволяют приблизиться к промежуточному случаю, когда $l \sim a$. Наше исследование является существенным обобщением представленных в работах [4], [7], [10] формул для функций Грина.

Точечный контакт моделируется круговым отверстием радиуса а, проделанным в непроницаемой мембране, которая разделяет пространство на две половины - левую L и правую R, каждая из которых занята однодоменным ферромагнитным металлом. Ось $z$ цилиндрической системы координат направлена перпендикулярно плоскости мембраны и проведена через центр отверстия (см. рис. 1).

\section{3. ОБЩЕЕ РЕШЕНИЕ ДЛЯ ФУНКЦИИ ГРИНА С УЧЕТОМ ГРАДИЕНТНЫХ СЛАГАЕМЫХ}

Решение системы (1) в координатном представлении при выбранной здесь системе единиц $k_{\mathrm{B}}=\hbar=1$ можно искать в виде

$$
g_{\mathrm{s}, \alpha}(z, \boldsymbol{\rho}, t)=\operatorname{th}\left(\frac{\varepsilon}{2 T}\right)+f_{\mathrm{s}, \alpha}(z, \boldsymbol{\rho}, t) .
$$

Первое слагаемое соответствует равновесной функции Грина $g_{\mathrm{s}}^{\mathrm{eq}}(\varepsilon)$ вдали от точки контакта, $\varepsilon$ - энергия электрона, $T$ - температура (см. обзор [1]). Второе слагаемое соответствует неравновесной части функции Грина, которая определяет неоднородность потока электронов в плоскости контакта. Однако систему уравнений (1) с учетом граничных условий (4) и (5) удобно решать в смешанном представлении, т. е. в координатном представлении по переменной $z$, в импульсном $k$-представлении по переменной $\rho$ и в энергетическом $\varepsilon$-представлении по переменной $t$. Кроме того, отметим, что далее в формулах мы не будем использовать спиновый индекс $\alpha$, так как в этом нет необходимости. Все последующие решения уравнений и выводы формул мы будем выполнять только для одной проекции спина электрона проводимости. При этом предполагается, что при прохождении контакта спин электрона не переворачивается. Итак, переходя в системе (1) к обратному преобразованию Фурье, получим

$$
\begin{aligned}
& l_{z} \frac{\partial g_{\mathrm{a}}(z, \mathbf{k}, \varepsilon)}{\partial z}+\left(1-i \mathbf{k} \cdot \mathbf{l}_{\|}\right) g_{\mathrm{s}}(z, \mathbf{k}, \varepsilon)=\left\langle g_{\mathrm{s}}\right\rangle \\
& l_{z} \frac{\partial g_{\mathrm{s}}(z, \mathbf{k}, \varepsilon)}{\partial z}+\left(1-i \mathbf{k} \cdot \mathbf{l}_{\|}\right) g_{\mathrm{a}}(z, \mathbf{k}, \varepsilon)=0 .
\end{aligned}
$$


Из уравнения (9) получаем связь симметричной и антисимметричной функций Грина:

$$
\frac{\partial g_{\mathrm{s}}(z, \mathbf{k}, \varepsilon)}{\partial z}=-\kappa g_{\mathrm{a}}(z, \mathbf{k}, \varepsilon)
$$

где для удобства введено обозначение $\kappa=\left[1-i\left(\mathbf{k} \cdot \mathbf{l}_{\|}\right)\right] / l_{z}$. Подставляя теперь (10) в уравнение (8), получим неоднородное дифференциальное уравнение второго порядка:

$$
\frac{\partial^{2} g_{\mathrm{s}}(z, \mathbf{k}, \varepsilon)}{\partial z^{2}}-\kappa^{2} g_{\mathrm{s}}(z, \mathbf{k}, \varepsilon)+\frac{\kappa}{l_{z}}\left\langle g_{\mathrm{s}}\right\rangle=0 .
$$

Общее решение этого уравнения можно представить в виде

$$
g_{\mathrm{s}}(z)=C_{1} e^{\kappa z}+C_{2} e^{-\kappa z}+\frac{1}{2 l_{z}}\left[\int_{\xi_{1}}^{z} e^{\kappa(\xi-z)}\left\langle g_{\mathrm{s}}(\xi)\right\rangle d \xi+\int_{z}^{\xi_{2}} e^{\kappa(z-\xi)}\left\langle g_{\mathrm{s}}(\xi)\right\rangle d \xi\right],
$$

где аргументы $\mathbf{k}$ и $\varepsilon$ функции Грина для простоты записи опущены. Далее оставим только те решения, которые имеют физический смысл. В области $z>0$, функция $g_{\mathrm{s}}(z)$ при $z \rightarrow \infty$ не должна расти до бесконечности (для конечного решения), следовательно, коэффициент $C_{1}$ равен нулю. Кроме того, при $\xi_{1}=0$ и $\xi_{2}=\infty$ имеем

$$
\left.g_{\mathrm{s}}(z)\right|_{z>0}=C_{2} e^{-\kappa z}+\frac{1}{2 l_{z}}\left[\int_{0}^{z} e^{\kappa(\xi-z)}\left\langle g_{\mathrm{s}}(\xi)\right\rangle d \xi+\int_{z}^{\infty} e^{\kappa(z-\xi)}\left\langle g_{\mathrm{s}}(\xi)\right\rangle d \xi\right] .
$$

Аналогично для области $z<0$ в формуле (12) коэффициент $C_{2}$ равен нулю. Используя пределы интегрирования $\xi_{1}=-\infty$ и $\xi_{2}=0$, получаем

$$
\left.g_{\mathrm{s}}(z)\right|_{z<0}=C_{1} e^{\kappa z}+\frac{1}{2 l_{z}}\left[\int_{-\infty}^{z} e^{\kappa(\xi-z)}\left\langle g_{\mathrm{s}}(\xi)\right\rangle d \xi+\int_{z}^{0} e^{\kappa(z-\xi)}\left\langle g_{\mathrm{s}}(\xi)\right\rangle d \xi\right] .
$$

Теперь продифференцируем по z полученные решения (13) и (14). Далее, умножив результат на множитель $-1 / \kappa$ и воспользовавшись соотношением (10), получаем интегральную связь антисимметричной и симметричной функций Грина:

$$
\begin{aligned}
& \left.g_{\mathrm{a}}(z)\right|_{z>0}=C_{2} e^{-\kappa z}+\frac{1}{2 l_{z}}\left[\int_{0}^{z} e^{\kappa(\xi-z)}\left\langle g_{\mathrm{s}}(\xi)\right\rangle d \xi-\int_{z}^{\infty} e^{\kappa(z-\xi)}\left\langle g_{\mathrm{s}}(\xi)\right\rangle d \xi\right] \\
& \left.g_{\mathrm{a}}(z)\right|_{z<0}=-C_{1} e^{\kappa z}+\frac{1}{2 l_{z}}\left[\int_{-\infty}^{z} e^{\kappa(\xi-z)}\left\langle g_{\mathrm{s}}(\xi)\right\rangle d \xi-\int_{z}^{0} e^{\kappa(z-\xi)}\left\langle g_{\mathrm{s}}(\xi)\right\rangle d \xi\right] .
\end{aligned}
$$

Выражая из (15) слагаемые $C_{1} e^{\kappa z}, C_{2} e^{-\kappa z}$ и подставляя их в (13) и (14), получаем

$$
\begin{aligned}
& \left.g_{\mathrm{s}}(z)\right|_{z>0}=\left.g_{\mathrm{a}}(z)\right|_{z>0}+\frac{1}{l_{z}} \int_{z}^{\infty} e^{-\kappa(\xi-z)}\left\langle g_{\mathrm{s}}(\xi)\right\rangle_{\mathrm{R}} d \xi \\
& \left.g_{\mathrm{s}}(z)\right|_{z<0}=-\left.g_{\mathrm{a}}(z)\right|_{z<0}+\frac{1}{l_{z}} \int_{-\infty}^{z} e^{-\kappa(z-\xi)}\left\langle g_{\mathrm{s}}(\xi)\right\rangle_{\mathrm{L}} d \xi
\end{aligned}
$$

Здесь важно отметить, что при определении области $z>0$ или $z<0$ усреднение $\left\langle g_{\mathrm{s}}(\xi)\right\rangle_{c}$, стоящее под знаком интеграла, соответствует усреднению по углам 
соответствующей стороны точечного контакта $c=(\mathrm{L}, \mathrm{R})$. На основании принятого основного решения (7) системы уравнений (8), (9) в стационарном случае имеем равенство

$$
\left.g_{\mathrm{s}}(z, \mathbf{k}, t)\right|_{t=0}=\left.f_{\mathrm{s}}(z, \mathbf{k}, t)\right|_{t=0},
$$

поэтому для удобства введем новые обозначения для симметричной и антисимметричной функций Грина:

$$
\begin{array}{ll}
\left.f_{\mathrm{s}}(z, \mathbf{k}, \varepsilon)\right|_{z<0} \equiv f_{\mathrm{s}}^{\mathrm{L}}, & \left.g_{\mathrm{a}}(z, \mathbf{k}, \varepsilon)\right|_{z<0} \equiv g_{\mathrm{a}}^{\mathrm{L}}, \\
\left.f_{\mathrm{s}}(z, \mathbf{k}, \varepsilon)\right|_{z>0} \equiv f_{\mathrm{s}}^{\mathrm{R}}, & \left.g_{\mathrm{a}}(z, \mathbf{k}, \varepsilon)\right|_{z>0} \equiv g_{\mathrm{a}}^{\mathrm{R}} .
\end{array}
$$

Тогда решения (16) в новых обозначениях перепишутся в виде

$$
\begin{aligned}
& f_{\mathrm{s}}^{\mathrm{L}}=-g_{\mathrm{a}}^{\mathrm{L}}+\frac{1}{l_{z}^{\mathrm{L}}} \int_{-\infty}^{z} e^{-\kappa^{\mathrm{L}}(z-\xi)}\left\langle f_{\mathrm{s}}^{\mathrm{L}}(\xi)\right\rangle_{\mathrm{L}} d \xi, \\
& f_{\mathrm{s}}^{\mathrm{R}}=g_{\mathrm{a}}^{\mathrm{R}}+\frac{1}{l_{z}^{\mathrm{R}}} \int_{z}^{\infty} e^{-\kappa^{\mathrm{R}}(\xi-z)}\left\langle f_{\mathrm{s}}^{\mathrm{R}}(\xi)\right\rangle_{\mathrm{R}} d \xi .
\end{aligned}
$$

Для нахождения антисимметричных функций Грина в точке $z=0$, удовлетворяющих граничным условиям (4) и (5), получим самосогласованные решения уравнений (17). Для этого отметим следующее. Усредненные по телесному углу симметричные функции Грина фактически являются электрохимическими потенциалами, которые неоднородны вдоль траекторий, пересекающих интерфейс двух ферромагнитных металлов [8]. Неоднородность электрохимического потенциала на границе (см. рис. 1б) выражается градиентным слагаемым в разложении этой функции в ряд по переменной $\xi$. Кроме того, функции $\left\langle g_{\mathrm{a}}^{c}\right\rangle_{c}$ и $\left\langle f_{\mathrm{s}}^{c}(\xi)\right\rangle_{c}$ удовлетворяют диффузионному уравнению с длиной затухания, много большей длины свободного пробега $l_{z}^{c}$ (см., например, [11]). Тогда мы можем разложить $\left\langle f_{\mathrm{s}}^{c}(\xi)\right\rangle_{c}$ в ряд около точки $z$ и вынести из-под интегралов независимые от $\xi$ члены. Подставляя разложения

$$
\left\langle f_{\mathrm{S}}^{c}(\xi)\right\rangle_{c} \simeq\left\langle f_{\mathrm{s}}^{c}(z)\right\rangle_{c}+\left.(\xi-z) \frac{\partial\left\langle f_{\mathrm{s}}^{c}(\xi)\right\rangle_{c}}{\partial \xi}\right|_{\xi=z}
$$

в уравнения (17) и усредняя по телесным углам в каждом полупространстве, запишем результат для правой стороны контакта:

$$
\begin{aligned}
\left\langle f_{\mathrm{s}}^{\mathrm{R}}(z)\right\rangle_{\mathrm{R}}= & \left\langle g_{\mathrm{a}}^{\mathrm{R}}\right\rangle_{\mathrm{R}}+\left\langle f_{\mathrm{s}}^{\mathrm{R}}(z)\right\rangle_{\mathrm{R}} \int_{z}^{\infty}\left\langle\frac{e^{-\kappa^{\mathrm{R}}(\xi-z)}}{l_{z}^{\mathrm{R}}}\right\rangle_{\mathrm{R}} d \xi+ \\
& +\left.\frac{\partial\left\langle f_{\mathrm{s}}^{\mathrm{R}}(\xi)\right\rangle_{\mathrm{R}}}{\partial \xi}\right|_{\xi=z} \int_{z}^{\infty}\left\langle\frac{e^{-\kappa^{\mathrm{R}}(\xi-z)}}{l_{z}^{\mathrm{R}}}\right\rangle_{\mathrm{R}}(\xi-z) d \xi .
\end{aligned}
$$

Далее введем новую независимую переменную $\eta \equiv \xi-z$ и перепишем это уравнение в точке $z \rightarrow 0$ :

$$
\begin{aligned}
\left\langle f_{\mathrm{s}}^{\mathrm{R}}(0)\right\rangle_{\mathrm{R}}= & \left\langle g_{\mathrm{a}}^{\mathrm{R}}\right\rangle_{\mathrm{R}}+\left\langle f_{\mathrm{s}}^{\mathrm{R}}(0)\right\rangle_{\mathrm{R}} \int_{0}^{\infty}\left\langle\frac{e^{-\kappa^{\mathrm{R}} \eta}}{l_{z}^{\mathrm{R}}}\right\rangle_{\mathrm{R}} d \eta+ \\
& +\left.\frac{\partial\left\langle f_{\mathrm{s}}^{\mathrm{R}}(\xi)\right\rangle_{\mathrm{R}}}{\partial \xi}\right|_{\xi=0} \int_{0}^{\infty}\left\langle\frac{e^{-\kappa^{\mathrm{R}} \eta}}{l_{z}^{\mathrm{R}}}\right\rangle_{\mathrm{R}} \eta d \eta
\end{aligned}
$$


Аналогичную процедуру проделаем с функцией Грина левой стороны контакта: введем новую независимую переменную $\eta \rightarrow-\eta \equiv z-\xi$ и сменим пределы интегрирования. Результат преобразований в формулах (17) запишем единой формулой:

$$
\begin{aligned}
\left\langle f_{\mathrm{s}}^{c}(0)\right\rangle_{c}= & \mp\left\langle g_{\mathrm{a}}^{c}\right\rangle_{c}+\left\langle f_{\mathrm{s}}^{c}(0)\right\rangle_{c} \int_{0}^{\infty}\left\langle\frac{e^{-\kappa^{c} \eta}}{l_{z}^{c}}\right\rangle_{c} d \eta \mp \\
& \left.\mp \frac{\partial\left\langle f_{\mathrm{s}}^{c}(\xi)\right\rangle_{c}}{\partial \xi}\right|_{\xi=0} \int_{0}^{\infty}\left\langle\frac{e^{-\kappa^{c} \eta}}{l_{z}^{c}}\right\rangle_{c} \eta d \eta,
\end{aligned}
$$

где знак минус соответствует функции Грина левой стороны контакта, а знак плюс функции Грина правой стороны. В выражении (21) все интегралы имеют аналитическое решение и выражаются в виде функциональных зависимостей от величины волнового вектора $k$ и спин-поляризованных длин свободного пробега $l_{z, \alpha}^{\mathrm{L}(\mathrm{R})}$. Подробное вычисление интегралов в (21) выходит за рамки нашей основной задачи, поэтому мы представим их как параметры и обозначим следующим образом:

$$
\lambda_{0}^{c}=\int_{0}^{\infty}\left\langle\frac{e^{-\kappa^{c} \eta}}{l_{z}^{c}}\right\rangle_{c} d \eta, \quad \lambda_{1}^{c}=\int_{0}^{\infty}\left\langle\frac{e^{-\kappa^{c} \eta}}{l_{z}^{c}}\right\rangle_{c} \eta d \eta .
$$

Тогда два линейных самосогласованных уравнения (21) можно записать в виде

$$
\left\langle f_{\mathrm{s}}^{c}\right\rangle_{c}=\mp \Lambda_{c}\left\langle g_{\mathrm{a}}^{c}\right\rangle_{c} \mp l^{c} \Lambda_{c}^{\prime} \frac{\partial\left\langle f_{\mathrm{s}}^{c}\right\rangle_{c}}{\partial \xi},
$$

где введены дополнительные обозначения отношения интегралов (22):

$$
\Lambda_{c} \equiv \frac{1}{1-\lambda_{0}^{c}}, \quad \Lambda_{c}^{\prime} \equiv \frac{\lambda_{1}^{c}}{l^{c}\left(1-\lambda_{0}^{c}\right)} .
$$

Итак, теперь мы можем записать окончательные выражения для симметричных функций Грина с учетом градиентных слагаемых. Для этого подставим усредненные выражения (23) в уравнения (17) и, переходя в этих уравнениях к переменным интегрирования $\eta= \pm(z-\xi)$, получим

$$
f_{\mathrm{s}}^{c}=\mp g_{\mathrm{a}}^{c} \mp \Lambda_{c}\left\langle g_{\mathrm{a}}^{c}\right\rangle_{c} \int_{0}^{\infty} \frac{e^{-\kappa^{c} \eta}}{l_{z}^{c}} d \eta \mp l^{c} \Lambda_{c}^{\prime} \frac{\partial\left\langle f_{\mathrm{s}}^{c}\right\rangle_{c}}{\partial \xi} \int_{0}^{\infty} \frac{e^{-\kappa^{c} \eta}}{l_{z}^{c}} d \eta,
$$

где знак минус соответствует функции Грина левой стороны контакта, а знак плюсфункции Грина правой стороны.

Теперь вычислим антисимметричные функции Грина $g_{\mathrm{a}}^{c}$. Для этого подставим полученный результат (25) в граничные условия (4) и (5). Однако предварительно запишем граничное условие (5), используя обратное преобразование Фурье:

$$
2 R g_{\mathrm{a}}(0, \mathbf{k}, \varepsilon)=-D\left[g_{\mathrm{s}}^{\mathrm{R}}(0, \mathbf{k}, \varepsilon)-g_{\mathrm{s}}^{\mathrm{L}}(0, \mathbf{k}, \varepsilon)\right]
$$

Здесь на основании (7) и преобразования Фурье симметричные функции Грина имеют вид

$$
\begin{aligned}
g_{\mathrm{s}}^{\mathrm{R}}(0, \mathbf{k}, \varepsilon) & =\frac{2 \pi a}{k} J_{1}(k a) \operatorname{th}\left(\frac{\varepsilon}{2 T}\right)+f_{\mathrm{s}}^{\mathrm{R}}(0, \mathbf{k}, \varepsilon), \\
g_{\mathrm{s}}^{\mathrm{L}}(0, \mathbf{k}, \varepsilon) & =\frac{2 \pi a}{k} J_{1}(k a) \operatorname{th}\left(\frac{\varepsilon-e V}{2 T}\right)+f_{\mathrm{s}}^{\mathrm{L}}(0, \mathbf{k}, \varepsilon),
\end{aligned}
$$


где $J_{1}(k a)$ - функция Бесселя первого порядка, $k$ - абсолютная величина волнового вектора в плоскости контакта, $V$ - приложенное напряжение к берегам контакта. Подставляя $(27)$ в $(26)$, затем используя условие $R+D=1$, получаем

$$
\begin{aligned}
(1-D) g_{\mathrm{a}}(0, \mathbf{k}, \varepsilon)=- & D \frac{\pi a}{k} J_{1}(k a)\left[\operatorname{th}\left(\frac{\varepsilon}{2 T}\right)-\operatorname{th}\left(\frac{\varepsilon-e V}{2 T}\right)\right]- \\
& -\frac{D}{2}\left[f_{\mathrm{s}}^{\mathrm{R}}(0, \mathbf{k}, \varepsilon)-f_{\mathrm{s}}^{\mathrm{L}}(0, \mathbf{k}, \varepsilon)\right] .
\end{aligned}
$$

Далее, подставляя в эту формулу симметричные функции Грина (25) для каждой стороны контакта и учитывая граничные условия (4), получаем основное выражение для антисимметричной функции Грина:

$$
\begin{aligned}
g_{\mathrm{a}}(0, \mathbf{k}, \varepsilon)=- & D \frac{\pi a}{k} J_{1}(k a)\left[\operatorname{th}\left(\frac{\varepsilon}{2 T}\right)-\operatorname{th}\left(\frac{\varepsilon-e V}{2 T}\right)\right]- \\
& -\Lambda_{\mathrm{L}}\left\langle g_{\mathrm{a}}^{\mathrm{L}}\right\rangle_{\mathrm{L}} \int_{0}^{\infty} \frac{D e^{-\kappa^{\mathrm{L}} \eta}}{2 l_{z}^{\mathrm{L}}} d \eta-l^{\mathrm{L}} \Lambda_{\mathrm{L}}^{\prime} \frac{\partial\left\langle f_{\mathrm{s}}^{\mathrm{L}}\right\rangle_{\mathrm{L}}}{d \xi} \int_{0}^{\infty} \frac{D e^{-\kappa^{\mathrm{L}} \eta}}{2 l_{z}^{\mathrm{L}}} d \eta- \\
& -\Lambda_{\mathrm{R}}\left\langle g_{\mathrm{a}}^{\mathrm{R}}\right\rangle_{\mathrm{R}} \int_{0}^{\infty} \frac{D e^{-\kappa^{\mathrm{R}} \eta}}{2 l_{z}^{\mathrm{R}}} d \eta-l^{\mathrm{R}} \Lambda_{\mathrm{R}}^{\prime} \frac{\partial\left\langle f_{\mathrm{s}}^{\mathrm{R}}\right\rangle_{\mathrm{R}}}{d \xi} \int_{0}^{\infty} \frac{D e^{-\kappa^{\mathrm{R}} \eta}}{2 l_{z}^{\mathrm{R}}} d \eta .
\end{aligned}
$$

Чтобы найти окончательное выражение для функции Грина $g_{\mathrm{a}}(0, \mathbf{k}, \varepsilon)$, мы усредним выражение (29) по телесным углам левой и правой сторон контакта и воспользуемся непрерывностью функции $g_{\mathrm{a}}(0, \mathbf{k}, \varepsilon)$ на границе (см. условие $\left.(4)\right)$.

В заключение этого раздела отметим, что в баллистическом режиме $(l>a)$ первое слагаемое в выражении (29) существенно превосходит остальные, поскольку в них большие значения длин свободного пробега $l_{z}^{\mathrm{L}(\mathrm{R})}$ стоят в знаменателях, так что в этом случае добавки от интегралов не приведут к большим ошибкам. Кроме того, надо отметить, что используемые нами приближения могут оказаться неприменимыми в промежуточном режиме, когда $l \sim a$, и значения этих величин составляют несколько ангстрем. Однако предложенная схема вычисления функций Грина может быть использована как некоторая интерполяционная процедура, приводящая к конечному результату.

\section{4. ФУНКЦИИ ГРИНА ТОЧЕЧНОГО КОНТАКТА БЕЗ УЧЕТА ГРАДИЕНТНЫХ СЛАГАЕМЫХ}

Сначала найдем выражение для антисимметричной функции Грина без учета градиентов. В этом случае соотношение (29) запишется в виде

$$
g_{\mathrm{a}}(0, \mathbf{k}, \varepsilon)=-D \gamma_{k}-\Lambda_{\mathrm{L}}\left\langle g_{\mathrm{a}}^{\mathrm{L}}\right\rangle_{\mathrm{L}} \int_{0}^{\infty} \frac{D e^{-\kappa^{\mathrm{L}} \eta}}{2 l_{z}^{\mathrm{L}}} d \eta-\Lambda_{\mathrm{R}}\left\langle g_{\mathrm{a}}^{\mathrm{R}}\right\rangle_{\mathrm{R}} \int_{0}^{\infty} \frac{D e^{-\kappa^{\mathrm{R}} \eta}}{2 l_{z}^{\mathrm{R}}} d \eta,
$$

где для сокращения записи мы ввели дополнительное обозначение

$$
\gamma_{k}=\frac{\pi a}{k} J_{1}(k a)\left[\operatorname{th}\left(\frac{\varepsilon}{2 T}\right)-\operatorname{th}\left(\frac{\varepsilon-e V}{2 T}\right)\right] .
$$

Теперь, предполагая, что антисимметричная функция Грина $g_{\mathrm{a}}$ в левой части соотношения (30) согласно граничному условию (4) равна как $g_{\mathrm{a}}^{\mathrm{L}}$, так и $g_{\mathrm{a}}^{\mathrm{R}}$, после 
поочередного усреднения по телесному углу в соответствующем полупространстве получим систему двух уравнений

$$
\begin{aligned}
\left\langle g_{\mathrm{a}}^{\mathrm{L}}\right\rangle_{\mathrm{L}}=- & \langle D\rangle_{\mathrm{L}} \gamma_{k}-\Lambda_{\mathrm{L}}\left\langle g_{\mathrm{a}}^{\mathrm{L}}\right\rangle_{\mathrm{L}} \int_{0}^{\infty}\left\langle\frac{D e^{-\kappa^{\mathrm{L}} \eta}}{2 l_{z}^{\mathrm{L}}}\right\rangle_{\mathrm{L}} d \eta- \\
& -\Lambda_{\mathrm{R}}\left\langle g_{\mathrm{a}}^{\mathrm{R}}\right\rangle_{\mathrm{R}} \int_{0}^{\infty}\left\langle\frac{D e^{-\kappa^{\mathrm{R}} \eta}}{2 l_{z}^{\mathrm{R}}}\right\rangle_{\mathrm{L}} d \eta, \\
\left\langle g_{\mathrm{a}}^{\mathrm{R}}\right\rangle_{\mathrm{R}}=- & \langle D\rangle_{\mathrm{R}} \gamma_{k}-\Lambda_{\mathrm{L}}\left\langle g_{\mathrm{a}}^{\mathrm{L}}\right\rangle_{\mathrm{L}} \int_{0}^{\infty}\left\langle\frac{D e^{-\kappa^{\mathrm{L}} \eta}}{2 l_{z}^{\mathrm{L}}}\right\rangle_{\mathrm{R}} d \eta- \\
& -\Lambda_{\mathrm{R}}\left\langle g_{\mathrm{a}}^{\mathrm{R}}\right\rangle_{\mathrm{R}} \int_{0}^{\infty}\left\langle\frac{D e^{-\kappa^{\mathrm{R}} \eta}}{2 l_{z}^{\mathrm{R}}}\right\rangle_{\mathrm{R}} d \eta .
\end{aligned}
$$

Для упрощения этих формул введем обозначения интегралов

$$
\begin{aligned}
\lambda_{\mathrm{L}}^{\mathrm{L}} \equiv \int_{0}^{\infty}\left\langle\frac{D e^{-\kappa^{\mathrm{L}} \eta}}{2 l_{z}^{\mathrm{L}}}\right\rangle_{\mathrm{L}} d \eta, & \lambda_{\mathrm{L}}^{\mathrm{R}} \equiv \int_{0}^{\infty}\left\langle\frac{D e^{-\kappa^{\mathrm{R}} \eta}}{2 l_{z}^{\mathrm{R}}}\right\rangle_{\mathrm{L}} d \eta, \\
\lambda_{\mathrm{R}}^{\mathrm{L}} \equiv \int_{0}^{\infty}\left\langle\frac{D e^{-\kappa^{\mathrm{L}} \eta}}{2 l_{z}^{\mathrm{L}}}\right\rangle_{\mathrm{R}} d \eta, & \lambda_{\mathrm{R}}^{\mathrm{R}} \equiv \int_{0}^{\infty}\left\langle\frac{D e^{-\kappa^{\mathrm{R}} \eta}}{2 l_{z}^{\mathrm{R}}}\right\rangle_{\mathrm{R}} d \eta .
\end{aligned}
$$

Отметим, что вычисление этих интегралов не является нашей главной задачей. Их аналитические выражения с коэффициентом прохождения $D$ для вычисления магнитосопротивления точечного контакта с магнитной доменной стенкой приведены в работе [7]. Таким образом, используя обозначения (32), мы приводим систему уравнений (31) к достаточно простому виду:

$$
\begin{aligned}
\left\langle g_{\mathrm{a}}^{\mathrm{L}}\right\rangle_{\mathrm{L}} & =-\langle D\rangle_{\mathrm{L}} \gamma_{k}-\Lambda_{\mathrm{L}}\left\langle g_{\mathrm{a}}^{\mathrm{L}}\right\rangle_{\mathrm{L}} \lambda_{\mathrm{L}}^{\mathrm{L}}-\Lambda_{\mathrm{R}}\left\langle g_{\mathrm{a}}^{\mathrm{R}}\right\rangle_{\mathrm{R}} \lambda_{\mathrm{L}}^{\mathrm{R}}, \\
\left\langle g_{\mathrm{a}}^{\mathrm{R}}\right\rangle_{\mathrm{R}} & =-\langle D\rangle_{\mathrm{R}} \gamma_{k}-\Lambda_{\mathrm{L}}\left\langle g_{\mathrm{a}}^{\mathrm{L}}\right\rangle_{\mathrm{L}} \lambda_{\mathrm{R}}^{\mathrm{L}}-\Lambda_{\mathrm{R}}\left\langle g_{\mathrm{a}}^{\mathrm{R}}\right\rangle_{\mathrm{R}} \lambda_{\mathrm{R}}^{\mathrm{R}} .
\end{aligned}
$$

Решая эту систему, получаем усредненные выражения для антисимметричных функций Грина слева и справа от границы контакта:

$$
\begin{aligned}
\left\langle g_{\mathrm{a}}^{\mathrm{L}}\right\rangle_{\mathrm{L}} & =\frac{\left(1+\Lambda_{\mathrm{R}} \lambda_{\mathrm{R}}^{\mathrm{R}}\right)\langle D\rangle_{\mathrm{L}}-\Lambda_{\mathrm{R}} \lambda_{\mathrm{L}}^{\mathrm{R}}\langle D\rangle_{\mathrm{R}}}{\Lambda_{\mathrm{L}} \lambda_{\mathrm{R}}^{\mathrm{L}} \Lambda_{\mathrm{R}} \lambda_{\mathrm{L}}^{\mathrm{R}}-\left(1+\Lambda_{\mathrm{L}} \lambda_{\mathrm{L}}^{\mathrm{L}}\right)\left(1+\Lambda_{\mathrm{R}} \lambda_{\mathrm{R}}^{\mathrm{R}}\right)} \gamma_{k}, \\
\left\langle g_{\mathrm{a}}^{\mathrm{R}}\right\rangle_{\mathrm{R}} & =\frac{\left(1+\Lambda_{\mathrm{L}} \lambda_{\mathrm{L}}^{\mathrm{L}}\right)\langle D\rangle_{\mathrm{R}}-\Lambda_{\mathrm{L}} \lambda_{\mathrm{R}}^{\mathrm{L}}\langle D\rangle_{\mathrm{L}}}{\Lambda_{\mathrm{L}} \lambda_{\mathrm{R}}^{\mathrm{L}} \Lambda_{\mathrm{R}} \lambda_{\mathrm{L}}^{\mathrm{R}}-\left(1+\Lambda_{\mathrm{L}} \lambda_{\mathrm{L}}^{\mathrm{L}}\right)\left(1+\Lambda_{\mathrm{R}} \lambda_{\mathrm{R}}^{\mathrm{R}}\right)} \gamma_{k} .
\end{aligned}
$$

Подставляя решения (33) в уравнение (30), получаем

$$
g_{\mathrm{a}}(0, \mathbf{k}, \varepsilon)=-\gamma_{k}\left\{D-G_{\mathrm{L}} I_{\mathrm{L}}-G_{\mathrm{R}} I_{\mathrm{R}}\right\}
$$

где для компактности записи введены обозначения

$$
\begin{aligned}
G_{\mathrm{L}} & =\Lambda_{\mathrm{L}} \frac{\left(1+\Lambda_{\mathrm{R}} \lambda_{\mathrm{R}}^{\mathrm{R}}\right)\langle D\rangle_{\mathrm{L}}-\Lambda_{\mathrm{R}} \lambda_{\mathrm{L}}^{\mathrm{R}}\langle D\rangle_{\mathrm{R}}}{\left(1+\Lambda_{\mathrm{L}} \lambda_{\mathrm{L}}^{\mathrm{L}}\right)\left(1+\Lambda_{\mathrm{R}} \lambda_{\mathrm{R}}^{\mathrm{R}}\right)-\Lambda_{\mathrm{L}} \lambda_{\mathrm{R}}^{\mathrm{L}} \Lambda_{\mathrm{R}} \lambda_{\mathrm{L}}^{\mathrm{R}}}, \\
G_{\mathrm{R}} & =\Lambda_{\mathrm{R}} \frac{\left(1+\Lambda_{\mathrm{L}} \lambda_{\mathrm{L}}^{\mathrm{L}}\right)\langle D\rangle_{\mathrm{R}}-\Lambda_{\mathrm{L}} \lambda_{\mathrm{R}}^{\mathrm{L}}\langle D\rangle_{\mathrm{L}}}{\left(1+\Lambda_{\mathrm{L}} \lambda_{\mathrm{L}}^{\mathrm{L}}\right)\left(1+\Lambda_{\mathrm{R}} \lambda_{\mathrm{R}}^{\mathrm{R}}\right)-\Lambda_{\mathrm{L}} \lambda_{\mathrm{R}}^{\mathrm{L}} \Lambda_{\mathrm{R}} \lambda_{\mathrm{L}}^{\mathrm{R}}}, \\
I_{\mathrm{L}} & =\int_{0}^{\infty} \frac{D e^{-\kappa^{\mathrm{L}} \eta}}{2 l_{z}^{\mathrm{L}}} d \eta, \quad I_{\mathrm{R}}=\int_{0}^{\infty} \frac{D e^{-\kappa^{\mathrm{R}} \eta}}{2 l_{z}^{\mathrm{R}}} d \eta .
\end{aligned}
$$


Отсюда видно, что антисимметричную функцию Грина можно представить как функцию поперечного волнового вектора и энергии электрона на интерфейсе гетероструктуры, состоящей из нормальных и (или) ферромагнитных металлов, если известны коэффициенты прохождения спиновых каналов проводимости.

\section{5. ФУНКЦИИ ГРИНА ТОЧЕЧНОГО КОНТАКТА С УЧЕТОМ ГРАДИЕНТНЫХ СЛАГАЕМЫХ}

Теперь в уравнении (29) учтем градиентные слагаемые и воспользуемся усредненными производными симметричных функций Грина, которые равны антисимметричным функциям Грина (см. формулу (10)). Тогда, используя введенные ранее обозначения интегралов (37), запишем уравнение (29) в виде

$$
\begin{aligned}
g_{\mathrm{a}}(0, \mathbf{k}, \varepsilon)=- & D \gamma_{k}-\Lambda_{\mathrm{L}}\left\langle g_{\mathrm{a}}^{\mathrm{L}}\right\rangle_{\mathrm{L}} I_{\mathrm{L}}+l^{\mathrm{L}} \Lambda_{\mathrm{L}}^{\prime}\left\langle\kappa^{\mathrm{L}} g_{\mathrm{a}}^{\mathrm{L}}\right\rangle_{\mathrm{L}} I_{\mathrm{L}}- \\
& -\Lambda_{\mathrm{R}}\left\langle g_{\mathrm{a}}^{\mathrm{R}}\right\rangle_{\mathrm{R}} I_{\mathrm{R}}+l^{\mathrm{R}} \Lambda_{\mathrm{R}}^{\prime}\left\langle\kappa^{\mathrm{R}} g_{\mathrm{a}}^{\mathrm{R}}\right\rangle_{\mathrm{R}} I_{\mathrm{R}} .
\end{aligned}
$$

В этом выражении имеются средние величины $\left\langle g_{\mathrm{a}}^{c}\right\rangle_{c}$ и $\left\langle\kappa^{c} g_{\mathrm{a}}^{c}\right\rangle_{c}$, которые необходимо найти. Действуя, как и в случае расчета функций Грина без учета градиентов, выполним усреднение по левой и правой сторонам контакта. В этом случае получаются громоздкие, но простые системы уравнений относительно $\left\langle g_{\mathrm{a}}^{c}\right\rangle_{c}$ и $\left\langle\kappa^{c} g_{\mathrm{a}}^{c}\right\rangle_{c}$. Отметим, что средние величины $\left\langle\kappa^{c} g_{\mathrm{a}}^{c}\right\rangle_{c}$ будут также выражаться через интегралы типа $\left\langle\kappa^{c} I_{c^{\prime}}\right\rangle_{c}$ (где $c^{\prime}$ может не совпадать с $c$ ), которые можно вычислить. Решая системы сначала относительно $\left\langle g_{\mathrm{a}}^{c}\right\rangle_{c}$, а затем относительно $\left\langle\kappa^{c} g_{\mathrm{a}}^{c}\right\rangle_{c}$ и выделяя из решений множитель $\gamma_{k}$, получим

$$
\begin{aligned}
g_{\mathrm{a}}(0, \mathbf{k}, \varepsilon)=- & \frac{\pi a}{k} J_{1}(k a)\left[\operatorname{th}\left(\frac{\varepsilon}{2 T}\right)-\operatorname{th}\left(\frac{\varepsilon-e V}{2 T}\right)\right] \times \\
& \times\left\{D-G_{\mathrm{L}} I_{\mathrm{L}}-G_{\mathrm{R}} I_{\mathrm{R}}-F^{\mathrm{grad}}\left(\mathbf{k}, D, l_{z}^{\mathrm{L}}, l_{z}^{\mathrm{R}}\right)\right\},
\end{aligned}
$$

где $F^{\operatorname{grad}}\left(\mathbf{k}, D, l_{z}^{\mathrm{L}}, l_{z}^{\mathrm{R}}\right)$ представляет собой громоздкую функцию из нескольких слагаемых, введенных выше обозначений интегралов и средних величин от известных параметров задачи. Отметим, что здесь слагаемые в фигурных скобках являются функциями поперечного волнового вектора и скалярных параметров контакта, которые не зависят от энергии. Множитель $\left[\operatorname{th}\left(\frac{\varepsilon}{2 T}\right)-\operatorname{th}\left(\frac{\varepsilon-e V}{2 T}\right)\right]$ в фурье-представлении по энергии $\varepsilon$ в стационарном случае равен $2 e V$. Таким образом, окончательно с учетом спинового индекса антисимметричную функцию Грина мы запишем в виде

$$
g_{\mathrm{a}, \alpha}(\mathbf{k})=-\frac{2 \pi a e V}{k} J_{1}(k a)\left\{D_{\alpha}-F_{\alpha}^{\mathrm{heter}}(\mathbf{k})-F_{\alpha}^{\mathrm{grad}}(\mathbf{k})\right\},
$$

где слагаемое $F_{\alpha}^{\text {heter }}$ представляет собой сумму $G_{\mathrm{L}, \alpha} I_{\mathrm{L}, \alpha}+G_{\mathrm{R}, \alpha} I_{\mathrm{R}, \alpha}$ (см. (34)-(37)).

В заключение этого раздела отметим, что полученное выражение (40) для антисимметричной функции Грина позволяет численно рассчитать спин-зависимую электронную проводимость и магнитосопротивление в следующих структурах: в магнитных планарных контактах, при использовании только коэффициента прохождения $D_{\alpha}$ (см., например, [12]); в магнитных точечных гетероконтактах, при учете двух слагаемых $D_{\alpha}$ и $F_{\alpha}^{\text {heter }}(\mathbf{k})$ (см. [7]); в магнитных точечных гетероконтактах при исследовании более общего случая, когда $l \sim a$, с добавлением слагаемого $F_{\alpha}^{\operatorname{grad}}(\mathbf{k})($ см. [13]). 


\section{6. ЗАКЛЮЧЕНИЕ}

С помощью квазиклассических уравнений для функций Грина и граничных условий мы построили симметричную и антисимметричную функции Грина магнитного точечного гетероконтакта, в котором магнитные электроды могут быть разделены немагнитными металлическими или диэлектрическими слоями. Предложенная схема вычисления основывается на интерполяции слагаемых, входящих в функции Грина, в двух приближениях: диффузном $(l<a)$ и баллистическом $(l>a)$. Были учтены градиентные слагаемые в разложении функции Грина, которые позволяют приблизиться к промежуточному случаю, когда $l \sim a$, для объяснения многообразия наблюдаемых экспериментальных данных. Представленные теоретические выражения симметричных и антисимметричных функций Грина могут быть использованы при модельных расчетах спин-зависимой проводимости, гигантского и туннельного магнитосопротивления, а также интерпретации экспериментальных данных, полученных при исследовании резистивных свойств магнитных гетероконтактов и наноконтактов, таких как $\mathrm{Co} / \mathrm{Fe}, \mathrm{Ni} /$ Мю-металл и туннельных точечных контактов из $\mathrm{CoFeB} / \mathrm{MgO} / \mathrm{CoFe}$.

Благодарности. Автор благодарит доцента Р.Г. Деминова и профессора Л. Р. Тагирова за обсуждение результатов. Работа выполнена при финансовой поддержке РФФИ (грант № 14-02-00348а) и Программы повышения конкурентоспособности Казанского федерального университета, финансируемой правительством РФ.

\section{Список литературы}

[1] J. Rammer, H. Smith, Rev. Modern Phys., 58:2 (1986), 323-359.

[2] А. В. Зайцев, ЖЭТФ, 86:5 (1984), 1742-1758.

[3] C. Heide, R. Elliott, N. S. Wingreen, Phys. Rev. B, 59:6 (1999), 4287-4304.

[4] L. R. Tagirov, B. P. Vodopyanov, K. B. Efetov, Phys. Rev. B, 63:10 (2001), 104428, 4 pp., arXiv: cond-mat/0207648.

[5] M. Ye. Zhuravlev, H. O. Lutz, A. V. Vedyayev, Phys. Rev. B, 63:17 (2001), 174409, 7 pp.

[6] M. Ye. Zhuravlev, E. Y. Tsymbal, S. S. Jaswal, A. V. Vedyayev, B. Dieny, Appl. Phys. Lett., 83:17 (2003), 3534-3536.

[7] A. N. Useinov, R. G. Deminov, L.R. Tagirov, G. Pan, J. Phys.: Condens. Matter, 19:19 (2007), 196215, 10 pp., arXiv: 0706.0266.

[8] L. R. Tagirov, N. García, Superlattices and Microstructures, 41:2-3 (2007), 152-162.

[9] Н. Х. Усеинов, ФTT, 51:3 (2009), 508-513.

[10] А.Н. Усеинов, Л. Р. Тагиров, Р. Г. Дёминов, Н. Х. Усеинов, Ученые записки Казанск. гос. ун-та. Сер. Физ.-матем. науки, 149:3 (2007), 5-30.

[11] Л.Р. Тагиров, Б.П. Водопьянов, Р. Г. Дёминов, А.Н. Усеинов, Ученые записки Казанск. гос. ун-та. Сер. Физ.-матем. науки, 147:2 (2005), 42-51.

[12] A. N. Useinov, J. Kosel, N. Kh. Useinov, L. R. Tagirov, Phys. Rev. B., 84:8 (2011), 085424, 8 pp.

[13] R. G. Deminov, N. Kh. Useinov, L. R. Tagirov, Magnetic Resonance in Solids. Electron. J., 16:2 (2014), 14209, 9 pp. 